\title{
OPTIMIZACIÓN DEL CONSUMO ESPECÍFICO DE ENERGÍA EN EL PROCESO DE BENEFICIADO EN SECO DE VARIEDADES AMARGAS DE QUINUA, BASADO EN LA APLICACIÓN DE UN LECHO FLUIDIZADO DE TIPO SURTIDOR (LFTS)
}

\author{
Mauricio Obando Estrada, Ramiro Escalera Vásquez, Carla Quiroga Ledezma y Luis Arteaga Weill
}

\section{RESUMEN}

Se ha minimizado el consumo específico de energía para el novedoso proceso de beneficiado en seco de quinua, mediante el empleo de un lecho fluidizado de tipo surtidor (LFTS). Se estudiaron las condiciones de operación y características básicas de funcionamiento del lecho, en la remoción de saponinas de la quinua real blanca, proveniente de zonas productivas en los departamentos de Oruro y Potosí.

Se utilizaron dos reactores de vidrio a escala laboratorio de 7,5 y $20 \mathrm{~cm}$ de diámetro, boquillas de 1,4 a $5 \mathrm{~mm}$ de diámetro y alturas de lecho estático entre 12,5 y $17,5 \mathrm{~cm}$. Los reactores de laboratorio se alimentaron de aire utilizando un compresor de $400 \mathrm{Lmin}^{-1}$ de capacidad, provisto de dos medidores de flujo de 10-280 $\mathrm{Lmin}^{-1}$ y de un filtro de humedad y aceite. Muestras de quinua real blanca fueron procesadas en estos equipos de acuerdo a un diseño experimental, evaluándose el efecto de los factores: diámetro de reactor, diámetro de boquilla, altura de lecho y tiempo de proceso sobre el consumo específico de energía, el porcentaje de remoción de saponinas y el porcentaje de pérdida de masa.

Los factores más preponderantes sobre el consumo específico de energía son: el diámetro de boquilla, diámetro de lecho, tiempo de proceso y altura de lecho, lográndose valores de consumo específico de energía mínimos $(0,23 \mathrm{kWh}$ $\mathrm{kg}^{-1}$ ) con la siguiente combinación: diámetro de boquilla $3 \mathrm{~mm}$, diámetro de lecho $20 \mathrm{~cm}$, altura de lecho de $12,5 \mathrm{~cm}$ y 60 min de tiempo. En estas condiciones se obtuvo un valor próximo a 0,01\% de saponinas y una pérdida de masa menor al 5\%. Estos valores son inferiores a los obtenidos en estudios anteriores.

Para calcular la caída de presión en el lecho fluidizado de tipo surtidor, se han obtenido nuevos valores de las constantes $K$ y $n$ de la ecuación de Lama: $K=9,2572$ y $n=0,3308$, para el lecho de 7,5 $\mathrm{cm}$ de diámetro y $K=12,8453$ y $n=$ 0,3451 para el de $20 \mathrm{~cm}$ El uso de estas constantes permitió calcular la caída de presión global del sistema con bastante aproximación, respecto de los valores experimentales obtenidos para diferentes diámetros de boquilla y alturas de lecho. En general, las caídas de presión globales, para esta configuración de LFTS, son pequeñas, comparadas con las observadas en el anterior estudio [11], lo cual permitiría utilizar sopladores más económicos, en lugar de compresores de más alto precio, aspecto que favorece la economía del proceso.

Palabras Clave: Quinua, Remoción de Saponinas, Lecho Fluidizado de Tipo Surtidor, Consumo de Energía. 